\section{The Rectum}

The rectum is a region peculiarly adapted to the use of seeds, whether the growth is within reach of the anus or can only be reached from the abdomen. In the former case the growth itself and the whole of the pelvic tissues round it can be easily infiltrated with seeds from two punctures in the perineum on either side of the midline. With the patient in the lithotomy position the needle of the introducer is inserted to one side of the tip of the coccyx and fifteen platinum seeds of 2 millicuries each are introduced in long columns so as to be deposited as uniformly as possible on that side, and the procedure is then repeated on the opposite side. In this way, with a posterior growth, a substantial barrier of seeds can be constructed in the hollow of the sacrum behind the rectum in order to irradiate both the base of the growth and the rectal glands. The infiltration of a growth on the anterior wall is a little more difficult on account of its greater mobility. If the growth is situated on a higher level it must be exposed by laparotomy and the seeds introduced across the peritoneal cavity. The introduction of the seeds is perfectly simple, and as asepsis can be strictly observed it is not accompanied by any special risk; but as in this region the growth has usually encircled the bowel and produced obstruction, a permanent colostomy is almost essential.

Carcinoma of the rectum is very sensitive to radium, and a localized tumour may disappear with great rapidity, while even in advanced cases the insertion of radium may make it possible to remove a growth previously inoperable. The only difficulty is that the rectal mucosa is itself highly sensitive to radiation, and great caution in dosage is therefore necessary, since an excessive dose may produce a severe proctitis. Even where a successful result has been obtained it is probably safer, if the patient will consent, to excise the rectum from the perineum three months later wherever this is possible, for in the present state of our knowledge it is impossible to be certain that some portion of the growth has not escaped destruction.

\section{Conclusion}

There can be no doubt that in radium we have a weapon of extraordinary power in the treatment of cancer, but we are still far from knowing all its possibilities or from being able to direct its energies to the best advantage. May I suggest tentatively a few directions in which progress may be made?

On the physical side we must aim at obtaining perfect uniformity of radiation so that the whole area involved will receive equal treatment. The obvious method would be to store a vast amount of radium in a single bomb, but to my mind such a method is too wasteful to be practicable. It seems to me that the method I have suggested of uniform infiltration is more likely to lead to practical results, since here the whole of the energy of the radium is utilized instead of a minute fraction. I am endeavouring to devise methods by which still smaller seeds can be produced, so that the whole volume may be literally peppered with minute foci. But here one is limited by the fact that a certain thickness of metal must be retained to form a screen, however small the content of the seed.

A tumour is affected solely by the portion of radiation it absorbs. One of the uses of rays of high penetrating power is to reach tumours at a depth without injury to the overlying tissues. If by some means we could increase the opacity of the tumour we should proportionately increase the efficacy of our radiation, and if at the same time we could render the skin transparent, we should have solved one of the most difficult problems in radiology. On the biochemical side it does not seem impossible that some method might be devised for sensitizing the tissue of a tumour, and I would point out that this is more likely to be effected by stimulating its growth than by reducing its vitality. There is something fascinating in the idea of stimulating the cells of a growth to more rapid division in order that they may meet with more certain destruction.

How subtle is the problem you will see from an experiment originated by $Z$ waardemaker, but repeated by Professor Dixon of Cambridge, who described it to me. You are aware that a frog's heart perfused with Ringer's solution can be kept alive and beating normally for several days. The solution contains a minute trace of potassium, an element with a small but definite radioactivity. If this trace of potassium is removed, the heart at once flags and soon ceases to beat. If, however, before it has stopped, a few milligrams of radium are brought into its neighbourhood it immediately resumes its normal pulsation. It would seem, indeed, that gamma rays are essential to our very existence, facilitating in some subtle way the chemical changes upon which our life depends.

On the clinical side I feel certain that much might be done to protect the normal tissues upon which, as I have shown you, we largely depend for success. A very short experience of radium will show, for example, the immense importance of protecting the skin, and I do not think that it is sufficiently recognized how great a protection it is to keep the skin soaking in ointment. Subjected to this treatment the skin will hardly react, even if enormous doses are introduced into the tissues.

Far more difficult is the problem of dissemination. Irradiation of the whole body would probably lead to the death of the patient, so that perhaps it is fortunate that it is at present impracticable. I am told that irradiation sufficiently general for our purpose may be found in unlimited quantity in even the closest nebulae, but so far no means of access to this source has been provided.

But there is one line of progress open to us all. There is no doubt whatever that the cure of cancer would be vastly simplified by early diagnosis and early treatment. Surely it is not too much to hope that as people realize that cancer can be cured in its early stages by radium, they will no longer hesitate from the dread of an operation to consult their doctor. Much will be done by investigators in their laboratories and by surgeons in hospitals, but it is the general practitioner, in the ordinary course of his work, who must teach the public that the cure of cancer is waiting if only they will ask for it in good time.

\section{THE ROLE OF THE GENERAL PIACTITIONER IN ANTE-NATAL WORK *}

\author{
BY
}

W. H. F. OXLEY, M.R.C.S., L.R.C.P. MEMBER OF THE BRITISII COLLEGE OF OBSTETRICIANS AND GYNAECOLOGISTS

The Departmental Committee on Maternal Mortality and Morbidity, after careful consideration of the subject for nearly two years, reported that a large number of maternal deaths could be prevented if all women had efficient ante-natal care, and it considered that it should, wherever practicable, be undertaken by the person who would be called in for any abnormality of labournamely, in most cases, the general practitioner. ${ }^{1}$ This recommendation is bearing early fruit, for the scheme adopted by the Cumberland County Council, which was published in the British Medical Journal of October 18th, 1930 (p. 649), provides that a general practitioner shall *A British Medical Association Lecture delivered to the Winchester Division. 
examine ante-natally every case attended by a midwife, either at a clinic or at his own surgery. I understand that in Hampshire the clinics are now to be staffed by general practitioners, although I believe that provision is not yet made for the examination to take place in the doctor's own surgery. This is rather a pity, as one of the most important parts of ante-natal care is the establishing of perfect confidence between doctor and patient; 'and where can this be done better than in the consulting room? Publicly provided ante-natal clinics have so far failed to produce any manifest effect upon maternal mortality, largely owing to want of co-operation with the general practitioner, and the latter must now prove what he can do in bringing about the desired effect.

\section{The Practical Working of a Ciminic}

Very important factors conducing to the success of antenatal care are: $(a)$ the patient must be made to realize early that a deep interest is being taken in her welfare; (b) she must obey in every detail the instructions given to her; $(c)$ these instructions must be reduced to their utmost simplicity. Without the full co-operation of the patient success will be impossible. A special leaflet of instructions is given to every mother at the hospital with which I am connected, and acts as a reminder of the advice given to her by the midwife in charge. It would be improved by the provision of a space on the back on which to enter the next appointment. If the patient receives the impression of protection, there will be no difficulty in getting her to attend; but if she misses an appointment she should be visited and the reason discovered for her absence.

The clinics are to be staffed by general practitioners and midwives, and it should be realized from the first that their duties are not antagonistic but complementary. There must be no divided responsibility; there must be a definite understanding of the work of each. It should be remembered that practically all midwives have gone through a training and passed an examination in their work, and have paid what is for most of them a fairly considerable fee. They are rightly jealous of the right to practise midwifery conferred upon them thirty years ago, and they are sensitive lest, now that a doctor is to be associated with them in the ante-natal care of all their booked cases, there may be a tendency to reduce them to the lower position of maternity nurse, without any separate responsibility at all. I am sure that none of us want that; the midwife is more fitted by nature to exercise the patience and the gentle care which are so essential to the well-being of every case, and has proved her value as a necessary member of the obstetric team. The well-trained midwife knows that she has to do with normal cases enly, and she is able to do this and also to recognize the early signs of abnormality. The better she is trained the oftener will she call the doctor, and she should be encouraged to take over as much of the routine work as possible. It is the half-trained woman who exceeds her duties. It will tend to the smooth working of the clinic if the midwife who has booked the case is given the duty. of examining and taking full particulars of her cases before bringing them before the doctor. She should fill up a record card. She should present her case to the doctor, who will find out whether she considers it a normal case or not, and will go over it with her, explaining any abnormality found, pointing out things she may have missed, and giving her definite instructions as to any treatment required. In most cases it will be her duty to carry this out, and the more responsibility is left to her the greater will her skill and interest in the work become. She should be able to test the urine, to recognize malpresentations, and, as pregnancy approaches its term, to determine the size of the foetal head in relation to the pelvis, and even to forecast the probable course of labour with some degree of accuracy. There is a danger of giving the midwife a false sense of security by telling her that there is " nothing wrong" with a case. Such statements should be always qualified with the words " at present," letting her clearly understand that the responsibility is still upon her to look out for abnormalities. The doctor will soon get to know those midwives he can trust, and will get a good deal of interest out of the work of training those whose knowledge is insuffisient.

Record cards should be filled up in duplicate for every case-one for the clinic and one for the midwife. Entries should be made on both at every visit of the patient, and the midwife must take her card to the confinement. Nothing is so annoying to the doctor when he is called for in an abnormality than ignorance of the ante-natal findings. The card we have used for many years measures 5 by 8 inches, small enough to go into the midwifery bag or pocket, and yet large enough for all necessary entries. The midwife fills up the front, and the doctor puts any observations he wishes to make on the back in red ink. A separate chart and record combined is kept for the confinement, and a brief note of the result of the confinement is made on the record card for permanent reference.

\section{The Principles of Ante-natai. Cire}

So much has been written lately upon ante-natal care that there is no need for me to go over the ground, but I would like to draw attention to the chapter on it in the interim report of the Departmental Committee on Maternal Mortality and Morbidity, and to the appendix on "Ante-natal clinics : their conduct and scope." Each doctor working on the staff should get a copy. Before discussing the common abnormalities met with during ante-natal care, I should like to stress the necessity of looking after the general health of the mother. In an analysis I recently made of the work of the East End Maternity Hospital in the last five years, it was found that six out of the seven mothers who died were seriously ill with some intercurrent affection before labour commenced, and the deduction was fairly drawn that any material reduction in the already low maternal death rate of the hospital was largely dependent upon an improvement in the general health of the mother. This is again an argument for the employment of the general practitioner in this work, for the most vigilant ante-natal clinic is bound to lose sight of some patients who fall ill during pregnancy; the expectant mother goes to her family doctor, who, if connected with the clinic, will have to consider the influence of the disease upon her pregnancy, and will be in a better position to obtain the help required.

Heart disease is very frequently met with in association with pregnancy. Ninety-six cases were picked out by the midwife in charge of our ante-natal department out of the 10,376 cases attended during the last five years. In the majority the disease was suspected by the midwife on account of the history of scarlet or rheumatic fever. No symptoms were complained of, compensation was good, and, provided the obstetric conditions were normal, these women passed through pregnancy and labour without requiring special care. A note of the condition was always made on the record card which accompanies the patient, so that she should not be allowed a long or difficult second stage. In others the extra strain of pregnancy had made manifest some latent heart trouble, the symptoms complained of being chiefly breathlessness, anxiety, and sleeplessness. They required rest for a certain time each day; but as most of our patients are working-class women with home duties, such an order could not be obeyed at home, and they were persuaded 
to come into hospital. The moderate cases respond remarkably well to treatment; only six were so ill as to require induction of labour before term. Irregularity of the heart is a sign which the midwife is told to report to the doctor at once. A practical point to be remembered is that in those mothers who are too ill to feed their babies belladonna plasters must not be applied by the midwife to the breasts, on account of the paralysing action of atropine upon the vagus, with consequent quickening of the heart's action. ${ }^{2}$

Influenza, pneumonia, and other acute diseases are not seen in the ordinary ante-natal clinic, but under the new regime the general practitioner will be expected to do his best to guide the patients through such illnesses, with an eye to the future labour. One woman we had lately did not let us know she was ill. She went to her own doctor, who prescribed but did not visit her, and in some way she was not missed when she failed to appear at the ante-natal department. She died after an easy labour, and post-mortem examination revealed malignant endocarditis, a disease which is frequently mistaken for influenza. This death was probably due to the divided responsibility, which we hope to avoid in future.

Purulent vaginitis and cervicitis is frequently met with (74 cases out of 10,000$)$ and may be gonorrhoeal, although in nearly every one it is an exacerbation of a simple cervicitis due to the hyperaemia of the parts during pregnancy. In any case it should be treated, and we have found douches useless for the purpose. The cervical canal should be thoroughly swabbed with a saturated solution of picric acid in spirit. If a great deal of softened tissue is present a Volkmann's spoon may be used to scrape it away; provided the internal os is not entered there is no danger of bringing on premature labour. This little operation can be done in a private house if a suitable light can be provided, and in many cases an anaesthetic is not required. The best speculum to use is a fairly long Sims, the Auvard as usually made not being long enough to reach into the posterior fornix in pregnancy, together with the very useful anterior speculum designed by Remington Hobbs. The cervix should be drawn down with tissue forceps, as varicose veins, which bleed if wounded with a vulsellum forceps, are often present on the cervix during pregnancy, and so obscure the view of the external os. Pain is not caused if the cervix is not pulled sideways, and if the well-lubricated specula are not pressed upon the sensitive vestibule. Having treated the cervix, slowly withdraw the specula, swabbing the vagina as the rugae are smoothed out in front of them. The patient should lie down for an hour afterwards. One of the midwives attached to the service can be taught to hold the specula while the doctor does the swabbing, and during the following week she should give a daily simple douche.

Pyelonephritis is another common condition requiring treatment during pregnancy, and should always be suspected when a patient from the sixth month onwards has a rise of temperature with a pain in the right side. Pus and $B$. coli communis are found in the urine, and this condition is frequently missed by the midwife. The patient needs rest in bed, copious barley water, and aperients. The treatment we have found most successful is the changing of the reaction of the urine. It is usually acid, and a mixture containing a large dose of sodium citrate is given; at the end of a week or so the urine is again turned acid by the exhibition of hexamine with sodium acid phosphate.

Syphilis should be suspected if there is a history of previous premature labours or stillbirths, and a Wassermann reaction test taken. Ten years ago this disease was extremely prevalent in East London, but now we rarely see a case. If treatment is begun somewhere about the twenty-fourth or twenty-sixth week of pregnancy it will be successful in nearly every case in procuring a healthy full-time child. This is one of the few conditions occurring in the course of ante-natal care where the help of an expert is necessary.

Dental treatment should be arranged for. There is no evidence that this is likely to bring about miscarriage.

There is hardly need to mention constipation except to say that while it may be merely a discomfort in ordinary life, in pregnancy it may be a real danger; it is present in all severe cases of toxaemia. We find the most satisfactory treatment is by phenolphthalein and medical paraffin.

\section{Sipeial Abnormalities of Pregnincy}

Cases of toxaemia with hyperemesis are not very common, but are extremely serious. If they do not soon clear up under treatment, and in any case if the liver becomes affected, immediate emptying of the uterus is indicated. It is not worth while running risks, as the chances of a live baby are remote.

Albuminuria.-The possibility of the almost complete eradication of eclampsia by means of systematic examination of the urine during pregnancy, and drastic eliminative treatment, is now well recognized, but the treatment of it in the patient's home is most unsatisfactory, and so the case will be removed from the care of the general practitioner unless the authorities can be persuaded to allow him to take his part in the staffing of maternity hospitals. In hospital the treatment by starvation, purgation, and washing out the body by fluid is almost always successful in avoiding eclampsia. The patients drink freely of barley water, made palatable by a little lemon flavouring. They are given barley sugar in order to avoid acidosis, and after a day or two, as the amount of urine increases, and the albumin gets less, are put on to vegetable soups, fruit, spaghetti and tomatoes, and a variety of milk puddings. Too much milk does not appear to be good for them. On account of the danger of permanent damage to the kidneys, or death of the foetus, cases which do not clear up with two weeks of this treatment are induced, even though it may mean the birth of a non-viable child. The chance of the patient having a live child if she has had a serious condition of toxaemia before the twenty-eighth week is too remote to make it worth the risk, quite apart from the possibility of eclampsia. The number of cases admitted to hospital were 104 out of 10,000 .

Posterior positions of the vertex should be diagnosed by the midwife and brought to the attention of the doctor. These positions are the most troublesome cases with which the midwife or doctor has to deal.

The sequence of early rupture of membranes, slow dilatation of the cervix, exhaustion of the mother, death of the foetus, extraction by forceps, trauma, and death from sepsis, is all too common. Efforts should therefore be made to do rotation. Buist's binder has been used for this purpose at the hospital, but it is difficult to be dogmatic as to its effect, as a large number of cases diagnosed as posterior before the head is engaged rotate spontaneously, and when the head has engaged it has no effect. One is inclined to think, however, that if the head is late in engaging, either through disproportion or want of flexion, it does influence rotation. That is to say, it is effective in just those cases which are likely to be difficult, and should therefore be tried in all. We have certainly had fewer dangerous cases since it has been used systematically. It requires careful fixing by the doctor and daily adjusting by the midwife.

Breech presentations should also be diagnosed by the midwife. The danger of these to the child has been emphasized by Gibberd, who compiled the returns of six 
maternity hospitals and found that 30 per cent. were stillborn and 9 per cent. died before the fourteenth day. On account of these dangers, external cephalic version has been practised at the hospital for many years, and is usually performed at or about the thirty-fourth week of pregnancy. Later than this it is difficult and frequentiy impossible in primigravidae. In twin pregnancy it is usually impossible, and extension of the legs is another common cause of failure. In a few cases where repeated efforts have been made, there has been slight haemorrhage on the following day, and in others the umb.lical cord has been much twisted. If an experienced doctor and midwife were to be present at every breech delivery the foetal death rate could, of course, be very much reduced, and the necessity for turning be avoided, but under present circumstances there is no doubt that the lives of many children will be saved thereby. Although the Central Midwives Board rules permit a midwife to attend breech cases in primiparae, we always direct her to call a doctor on these occasions.

\section{Contracted Pelvis}

The diagnosis of contracted pelvis is simple, and can be made at any period during pregnancy, but there is no need to do so until about the thirty-fourth week, as treatment, whether by induction or Caesarean section, would not be taken until after then. The midwife should be instructed to suspect contracted pelvis if the mother is less than five feet in height. She should also take the external measurements of the pelvis-interspinous, intercristal, posterior interspinous, and external conjugate-and should call the doctor's attention to any deviation from the normal, and especially to all cases in which the external conjugate diameter measures 7 inches or less. It is as well for the doctor to check the midwife's results from time to time. In the early days of ante-natal care treatment was frequently based upon the result of these findings, but now the pendulum is swinging the other way, and the measurements are frequently said to be of no value. Both views are wrong. As a matter of fact, careful measurements do give some idea both as to the type of contraction and as to its degree. The mere anatomical fact of contraction being present is, however, not sufficient; we want to know much more than that. First, is the contraction of such a degree as to preclude absolutely the passage through the pelvis of a living child? Secondly, will the contraction interfere with the birth of a normal full-term child? Thirdly, will it interfere with the birth of the particular child which has to come through that individual pelvis? The third question is the important one. We have found that in at least 80 per cent. of cases in which there is a definite contraction, the normal course of labour is unaffected thereby. To diagnose and treat correctly the other 20 per cent. is where the difficulty comes in. What signs, other than the measurements, can be used as a guide; in other words, what influence has contraction of the pelvis to an obstetric degree upon the signs of pregnancy? In most cases nothing unusual will be found until the thirty-sixth week. At that time the upper pole of the foetus reaches the costal margin, and, after that, growth can take place only forwards, downwards, or sideways. If there is resistance to the usual downward growth a prominent abdomen or obliquity of the uterus will result. The former sign is the more common in multiparae, the latter in primiparae. Owing to the looseness of the abdominal muscles in a multipara, the head frequently does not engage in the pelvis until labour begins. In them the best guide to the probable course of labour is the previous history, although this is not infallible, owing to the increasing size of the child and weakness of the uterus with the increasing number of pregnancies. In a primipara, however, a head floating free above the brim at the end of the thirty-sixth week is an almost certain sign of contraction of the pelvis to an obstetric degree. The midwife should be taught to recognize these signs and to bring them to the notice of the doctor. In some cases, especially in flat pelves, the difficulty of engagement will cause the foetus to rotate on its long axis and become a breech, and in all breech presentations discovered during pregnancy this should be borne in mind. In many cases in which the head is found above the brim, it can be made to enter by pushing it downwards and backwards. In some cases, however, it is impossible to do this, the child's head bulging the lower part of the abdomen, and "overriding" the symphysis, and in these it is clear that a sufficient degree of dispropxtion already exists to interfere with the normal course of labour. Midwives will often inform the doctor that the head " pushes in," but it is well to beware of this statement, as in many cases in which they say it has done so the largest diameter of the head is still above the brim. Disregarding the few cases in which non-engagement is due to the presence of a tumour or of placenta praevia, it will be caused by occipito-posterior position or disproportion in size between head and pelvis, and a vaginal examination will be necessary. At this examination the diagonal conjugate will be measured, and, by sweeping the tip of the forefinger round the brim an idea as to the roominess at the sides can be obtained. If in a relaxed patient the promentory cannot be felt, it may be taken that the pelvis is normal. If the diagonal diameter measures 4 inches, induction at the thirty-sixth week will be necessary if the child is of normal size, and if less than 4 inches, Caesarean section will probably be the correct treatment.

In cases of slight contraction, with a diagonal conjugate of four and a quarter to four and a half inches, the question as to whether induction will be necessary at all, and, if it is, the decision as to the right time for performing it, is of the greatest difficulty. The answer depends upon so many factors, such as degree of overlapping, amount of room at the sides, condition of the mother, etc., that we are frequently finding that " experience is fallacious and judgement difficult." In these doubtful cases the best course is to prepare for induction, and to decide finally with the patient under deep anaesthesia. With the patient in the lithotomy position two fingers may be placed in the vagina, and the head pushed down upon them by the external hand, and a more accurate estimation of the relative size of head and pelvis thus be made. In a pelvis of the small round variety, the slightest degree of overlapping indicates immediate induction, but in a large flat pelvis with plenty of room at the sides slight overlapping will not prove an insuperable obstacle to the birth of a live child.

The operation of induction can be performed in a private house provided the two great difficulties-namely, (a) the obtaining of aseptic conditions, and (b) a good light_can be overcome. Krause's method has proved most successful with us. Large-sized bougies, about No. 13, are used; they are soaked for twenty-four hours in 1 in 1,000 perchloride of mercury and boiled for a minute immediately before use. The midwife can show the cervix in the way before described, and there is usually no difficulty in inserting the bougies at or after the thirty-sixth week, provided they are allowed time in stretching the internal os. In multiparae, and even in primiparae, who are not nervous, an anaesthetic is not necessary, if the help of a second midwife can be obtained.

It is a remarkable fact that in the last five years, during which 10,376 cases have been attended at the hospital, only one Caesarean section has been done, every other case of contracted pelvis having been treated by induction at or about the thirty-sixth week, or by the applica- 
tion of forceps. It seems, however, that many obstetric surgeons, imbued with what may be called tive "furor operativus," make the mere diagnosis of contracted pelvis a reason for the performance of this operation, apart altogether from other considerations. If cases were carefully considered on the lines given above, there is very little doubt that many unnecessary Caesarean sections would be avoided.

If I have gone rather minutely into detail, my excuse is that the more I think over the problem of maternal mortality the more convinced I become that it is only by close attention to every detail, and by co-operation between all the members of the obstetric team, and especially between general practitioners and midwives, that we shall make any real impression upon the number of women who are every year dying preventable deaths.

ReFERENCES

${ }^{1}$ Interim Report of Departmental Committee, para. 117

'Dixon: Pharinicology', 1923, p. 175.

\section{THE ACCESSORY LOBE OF THE AZYGOS VEIN}

BY

H. P. NELSON, M.B., F.R.C.S.

DEMONSTRATOR OF ANATOMY, ST. BARTHOLOMEW'S HOSPITAL AND

GEORGE SIMON, M.B., D.M.R.E.

CHIEF ASSISTANT, X-RAY DEPARTMINT, ST. BARTHOLOMEW'S HOSPITIL

The accessory pulmonary lobe which occurs at the apex of the right lung was first observed by the anatomist in 1778, but its radiographic appearances were not identified until 1923. Since radiographic examination has become the most fruitful method of investigation of the lungs it seems desirable to present a detailed account of the appearances of this infrequent pulmonary variation.

\section{Anatomy}

The accessory lobe of the azygos vein is an additional pulmonary lobe occurring at the right apex, and separated from the remainder of the apex by a fissure containing the vena azygos major. The vena azygos major pursues its usual course behind the oesophagus just to the right of the midline until it reaches the level of the sixth thoracic vertebra, when, instead of arching forwards over the root of the lung, it turns in a lateral direction, dipping into the upper lobe. There, after passing forwards and inclining medially, it terminates in the superior vena cava. The vein, contained in a complete pleural septum, separates the dome of the pleura into two compartments, with the result that an accessory lobe is split off from the medial side of the right upper lobe. This septum consists of both visceral and parietal layers of the pleura, and is so disposed that its outer surface is convex and the lower free margin, which contains between its layers the vena azygos, is arched. It thus forms a kind of meso-azygos (Fig. 1).

The anatomical descriptions of this abnormality are fairly unanimous, and the following account is based on the papers by Geddes, Holtby, and Stibbe; the last reviews all the cases that have appeared in the literature up to 1918. The position of the fissure, and in consequence the size of the accessory lobe, depend on the degree of lateral deviation of the vein. The fissure may split the outer surface of the upper lobe at a point 2 inches below the apex, and the fold will then lie more or less horizontal, the accessory lobe forming the greater part of the apex of the lung. On the other hand, the fissure may merely split off a small tongue-shaped lobe from the mediastinal surface of the lung. The average lies between these two extremes, the fissure usually descending from the apex of the luing. The level of termination of the azygos vein with tire vena cava superior is higher than normal, usually at the junction of the two innominate veins, and meets the lateral instead of the posterior surface of the vena cava. The depth of the fissure varies, but usually approximates to that of a primary lung fissure. In some of the described cases the vena azygos was resting on the eparterial bronchus, while in others there was as much as an inch of lung tissue between the deepest part of the fissure and the bronchus. The bronchus supplying the lobe comes from the eparterial: in three cases it has been described as having an $\mathrm{N}$-shape, first ascending into the lung on the outer side of the fissure and then down under the azygos vein to ascend into the accessory lobe.

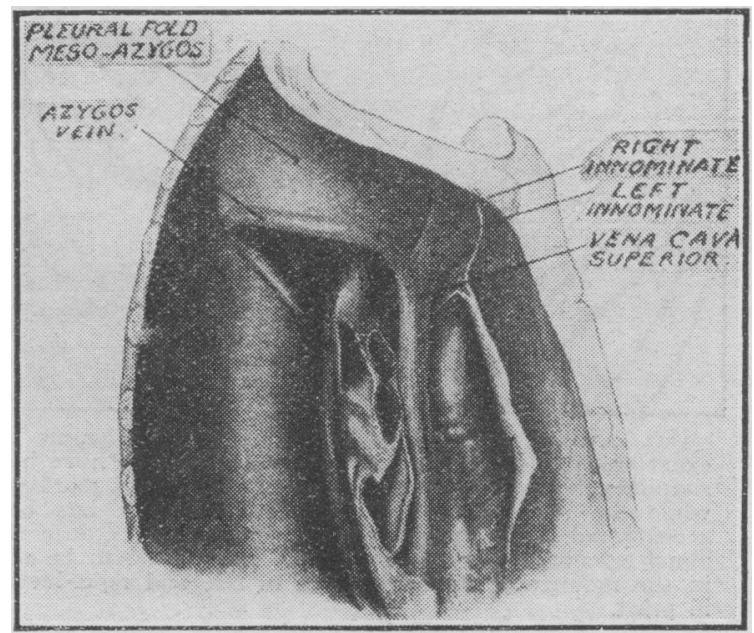

Fig. 1 (after Stibbe).-A case that has been dissected to show the mediastinal surface of the right pleural cavity after removal of the lung and accessory lobe. The pleural fold is shown with the vena azygos major running in its free margin.

In the development of this condition there are two variable factors: the growing lung buds and the growing cardinal veins. The posterior cardinal vein arches forwards across the coelom to join the duct of Cuvier, and it brings with it a fold of primitive pleural membrane, which divides the apex of the pleural-coelomic cavity into a medial and lateral compartment. The lung bud grows upwards and outwards, passing usually lateral to the vein, and with the descent of the heart the vein is drawn downwards and inwards and the medial apex obliterated. Now if there is a slight alteration in the position of either of these factors-a lung bud not lateral enough or the vein too lateral-the pleural fold would impinge against the lung and split off a medial process, which will expand upwards as the accessory lobe. Other accessory lobes, less common than the one here described, have occurred above and below the root of the lung. These other lobes have unfortunately been called " azygos lobes," and it is to distinguish it from these that the lobe we are concerned with has been called " the accessory lobe of the azygos vein." The fissure which separates these " azygos lobes" is lined only with visceral pleura, and is thus similar to a primary lung fissure, but the fissure which separates off the lobe of the azygos vein is lined by both visceral and parietal pleura.

\section{Radiography}

In 1923 Wessler and Jackes first drew attention to a falciform shadow near the right apex occasionally seen in antero-posterior radiograms of the chest. In 1928 Wessler and Bentick observed this shadow in two patients in whom later the opportunity occurred at post-mortem tc compare the anatomy of the lungs with the radiographic appearance. Both of these cases had an accessory lobe of the azygos vein, and they were thus able to prove beyond all doubt the cause of this shadow. 